\title{
Complete Genome Sequence Data of Xenorhabdus budapestensis Strain C72, a Candidate Biological Control Agent from China
}

\author{
Bo Li, ${ }^{1,2}$ Dewen Qiu, ${ }^{1}$ and Shuangchao Wang ${ }^{1, \dagger}$ \\ ${ }^{1}$ State Key Laboratory for Biology of Plant Diseases and Insect Pests, Institute of Plant Protection, \\ Chinese Academy of Agricultural Sciences, Beijing, China \\ ${ }^{2}$ Functional and Evolutionary Entomology, Gembloux Agro-Bio Tech, University of Liège, B-5030 \\ Gembloux, Belgium
}

\begin{abstract}
Xenorhabdus budapestensis strain C72 isolated from the entomopathogenic nematode of Steinernema bicornutum possesses an excellent biocontrol effect on southern corn leaf blight. However, its genomic information is lacking. Here, we report a high-quality complete and annotated genome sequence of $X$. budapestensis strain C72. Fifteen secondary metabolite biosynthetic gene clusters are identified in the genome, which are responsible for the production of a diverse group of antimicrobial compounds to help host plants against agricultural pathogenic diseases. This genome sequence could contribute to investigations of the molecular basis underlying the biocontrol activity of this Xenorhabdus strain.
\end{abstract}

\section{Resource Announcement}

The bacteria of genus Xenorhabdus belong to the family Enterobacteriaceae and establish obligate mutualistic associations with entomopathogenic nematodes of the genus Steinernema. They are well known for their potential to produce a variety of bioactive compounds with antimicrobial and insecticidal activities (Dreyer et al. 2018; Liu et al. 2000). In previous decades of research, the symbionts were commercially and successfully used as biological control agents in agriculture to kill a wide variety of pest insect species in China, North America, Europe, Australia, and Africa (Bera et al. 2014; Garriga et al. 2020). The symbiotic Xenorhabdus bacteria not only contribute to the nematode's ability to kill the host by releasing insecticidal proteins, immunosuppressors, and lytic enzymes but also restrain the growth of other microorganisms in the absence of nematodes by the production of antimicrobial compounds (Shi and Bode 2018).

Various kinds of biological molecules have been isolated and described from the genus Xenorhabdus. The main antimicrobial compounds include ribosomal-encoded benzylideneacetone (Ji et al. 2004), xenocin, and bicornutin (Böszörményi et al. 2009; Rathore 2013) and nonribosomally produced xenematides (Xi et al. 2019), fabclavines (Fuchs et al. 2014), nematophin (Li et al. 1997), xenocoumacin (Park et al. 2009), peptide-antimicrobial-Xenorhabdus lipopeptides (Fuchs et al. 2011), and rhabdopeptides (Zhao et al. 2018). Particular attention should be paid to some members of the genus Xenorhabdus such as Xenorhabdus budapestensis and X. nematophila for their antiphytopathogenic properties (Ji et al. 2004; Vozik et al. 2015); these species need to be comprehensively investigated for the possibility of using them as alternatives to chemical pesticides in agriculture.

${ }^{\dagger}$ Corresponding author: S. Wang; wangshuangchao@caas.cn

The author(s) declare no conflict of interest.
Funding

This work was financially supported by grants from the Agricultural Science and Technology Innovation Program of Chinese Academy of Agricultural Sciences (CAAS-2060302-051), the Elite Youth Program of the Chinese Academy of Agricultural Sciences, and a China Scholarship Council (201903250116) national scholarship for B. Li.

\section{Keywords}

biocontrol, genome, southern corn leaf blight, Xenorhabdus budapestensis 
Table 1. Identified secondary metabolite regions in genome of Xenorhabdus budapestensis C72 using antiSMASH 5.2.0

\begin{tabular}{|c|c|c|c|c|c|}
\hline \multirow{2}{*}{$\begin{array}{l}\text { Gene } \\
\text { cluster } \\
\text { Region } 1\end{array}$} & \multirow{2}{*}{$\begin{array}{l}\text { Most similar } \\
\text { known } \text { cluster }^{\mathbf{a}} \\
\text { Luminmide }\end{array}$} & \multirow{2}{*}{$\begin{array}{c}\text { Similarity } \\
\text { (\%) } \\
100\end{array}$} & \multirow{2}{*}{$\begin{array}{l}\text { Cluster types } \\
\text { NRPS }\end{array}$} & \multicolumn{2}{|c|}{$\begin{array}{c}\text { Gene sequence } \\
\text { locations }\end{array}$} \\
\hline & & & & 88,888 & 150,983 \\
\hline Region 2 & Safracin $A$ /safracin $B$ & 20 & NRPS-like & 414,262 & 454,892 \\
\hline Region 3 & Taxlllaid A & 4 & Lassopeptide & 696,101 & 718,483 \\
\hline Region 4 & $\begin{array}{l}\text { Xenocoumacin 1/ } \\
\text { xenocoumacin II }\end{array}$ & 14 & NRPS, T1PKS & $1,455,717$ & $1,532,527$ \\
\hline Region 5 & Fabclavine la & 100 & NRPS, T1PKS & $1,571,752$ & $1,691,805$ \\
\hline Region 6 & Yersiniabactin & 5 & NRPS, T1PKS & $2,003,086$ & $2,064,878$ \\
\hline Region 7 & Glidopeptin & 25 & NRPS & $2,164,883$ & $2,253,962$ \\
\hline Region 8 & Pyrrolizixenamide A & 100 & NRPS & $2,262,133$ & $2,307,370$ \\
\hline Region 9 & Nematophin & 37 & NRPS & $2,358,628$ & $2,412,306$ \\
\hline Region 10 & Neneprotides A-C & 55 & NRPS & $2,472,098$ & $2,585,011$ \\
\hline Region 11 & ND & & NRPS, AT-PKS & $2,630,477$ & $2,706,071$ \\
\hline Region 12 & $\begin{array}{l}\text { Xenoamicin A/ } \\
\text { xenoamicin B }\end{array}$ & 25 & NRPS & $2,716,887$ & $2,768,694$ \\
\hline Region 13 & ND & & Thiopeptide & $2,866,887$ & $2,892,869$ \\
\hline Region 14 & $\begin{array}{l}\text { Putrebactin/ } \\
\text { avaroferrin }\end{array}$ & 100 & Siderophore & $3,180,981$ & $3,205,638$ \\
\hline Region 15 & ND & & $\beta$-Lactone & $3,508,153$ & $3,533,766$ \\
\hline
\end{tabular}

${ }^{\mathrm{a}} \mathrm{ND}=$ not determined.

${ }^{\mathrm{b}} \mathrm{NRPS}=$ nonribosomal peptide synthetase and T1PKS = type I polyketide synthase.

We previously isolated and identified a strain $X$. budapestensis C72 from Steinernema bicornutum in Changbai Mountain, Jilin, China, which was considered a novel source of highly efficient antimicrobial compounds against plant pathogens. In our previous study, this strain possessed broad-spectrum antifungal activities in vitro and exhibited excellent biocontrol efficacy against Bipolaris maydis, which is the causal agent of southern corn leaf blight in the field (Li et al. 2021). It is also the first report of Xenorhabdus inhibiting B. maydis (Li et al. 2021). The antagonistic property of $X$. budapestensis $C 72$ is attributed to the production of bioactive metabolites (Iytic enzymes and antibiotics) and, hence, the isolate was molecularly and phylogenetically characterized (16S ribosomal RNA [rRNA] GenBank accession number MT967912.1) (Li et al. 2021). To date, only one draft genome sequence of $X$. budapestensis DSM 16342 strain has been reported (Tobias et al. 2017). Because very little is currently known about the beneficial properties of the $X$. budapestensis $\mathrm{sp}$., we sequenced the whole genome of the isolate $X$. budapestensis $C 72$ to further explore its genetic traits and biosynthetic gene clusters related to biologically active substances.

The $X$. budapestensis $\mathrm{C} 72$ strain was obtained by single-colony isolation on nutrient agar supplemented with bromothymol blue and triphenyl-2,3,5-tetrazolium chloride plates and was aerobically cultured in Luria-Bertani broth at $28^{\circ} \mathrm{C}$ for 2 days. Genomic DNA was extracted using a EasyPure Bacteria Genomic DNA Kit (TransGEN, Beijing, China), following the manufacturer's protocols, with RNase A treatment. The quality and quantity of the total DNA was evaluated via agarose gel electrophoresis and a Quant-iT PicoGreen dsDNA assay kit, respectively (Invitrogen, Carlsbad, CA, U.S.A.). Genomic DNA was sequenced and analyzed by OE Biotech Co., Ltd. (Shanghai, China). The sequencing libraries were prepared with the SMRTbell template prep kit 1.0 as directed by the manufacturer (Pacific Biosciences, Menlo Park, CA, U.S.A.), and single-molecule real-time (SMRT) sequencing was conducted on the PacBio Sequel platform. Default software settings were used throughout unless otherwise noted.

The high-quality filtered reads were assembled to generate one contig without gaps by using SMRT Analysis 2.3.0 (Pacific Bioscience), which yielded a total of 508,934 subreads $\left(\mathrm{N}_{50}\right.$ value $\left.=4,366,413\right)$ with an average length of $4,067 \mathrm{bp}$ (total $\left.=2,344,639,223 \mathrm{bp}\right)$, providing over 500-fold genome coverage. The size of the $X$. budapestensis $C 72$ genome was 4,366,413 bp with a $\mathrm{G}+\mathrm{C}$ content of $43.14 \%$. The genomic sequence was annotated by the NCBI Prokaryotic Genome Annotation Pipeline (v5.1) based on the Best-placed reference protein set and GeneMarkS+ (Tatusova et al. 2016). In all, 3,596 protein coding genes were predicted. Then, the transfer RNA (tRNA), rRNA, and small nuclear RNA (sRNA) were detected by tRNAscan-SE (v1.3.1) (Lowe and Eddy 1997), RNAmmer (v1.2) (Lagesen et al. 2007), and Rfam (v10.0) (Griffiths-Jones 2003), respectively. Among the total 104 noncoding RNA genes, 
22 genes encoded for rRNAs, 78 encoded for tRNAs, and 4 were identified as sRNAs. In total, 11 prophage sequences were detected using PhiSpy (v2.3) (Akhter et al. 2012). Analysis of secondary metabolite biosynthetic gene clusters was done with antiSMASH (v5.2.0) (Blin et al. 2019). The biosynthetic clusters (15 clusters) and bioproducts (e.g., fabclavine la, pyrrolizixenamide $A$, and putrebactin) present in $X$. budapestensis $C 72$ were predicted and are shown in Table 1. The different types of secondary metabolite clusters such as nonribosomal peptide synthetase (NRPS), polyketide synthase (PKS), and siderophore presented in the C72 strain genome sequence may play a crucial role in antimicrobial activity.

The C72 strain genome is the first $X$. budapestensis complete genome reported from China. The C72 genome information will provide a valuable resource for future studies on the understanding of the molecular basis of both the application of biocontrol and symbiotic relationship between $X$. budapestensis and their host nematodes.

Data availability. The complete genome sequence for $\mathrm{C} 72$ has been deposited into GenBank under the accession number CP072455 (genome annotation is available at https://www. ncbi.nlm.nih.gov/nuccore/2021543890/). The BioProject and BioSample designations for this project are PRJNA625211 and SAMN14595202, respectively. Raw reads have been deposited into NCBI Sequencing Read Archive (SRA accession number SRR13921535) in association with BioProject PRJNA625211.

\section{Literature Cited}

Akhter, S., Aziz, R. K., and Edwards, R. A. 2012. PhiSpy: A novel algorithm for finding prophages in bacterial genomes that combines similarity- and composition-based strategies. Nucleic Acids Res. 40:e126.

Bera, T., Pandey, A. K., Maurya, R. P., and Mall, P. 2014. Role of entomopathogenic nematodes in the management of insect pests. J. Entomol. Res. 38:301-310.

Blin, K., Shaw, S., Steinke, K., Villebro, R., Ziemert, N., Lee, S. Y., Medema, M. H., and Weber, T. 2019. antiSMASH 5.0: Updates to the secondary metabolite genome mining pipeline. Nucleic Acids Res. 47:W81-W87.

Böszörményi, E., Érsek, T., Fodor, A., Fodor, A. M., Földes, L. S., Hevesi, M., Hogan, J. S., Katona, Z., Klein, M. G., Kormány, A., Pekár, S., Szentirmai, A., Sztaricskai, F., and Taylor, R. A. J. 2009. Isolation and activity of Xenorhabdus antimicrobial compounds against the plant pathogens Erwinia amylovora and Phytophthora nicotianae. J. Appl. Microbiol. 107:746-759.

Dreyer, J., Malan, A. P., and Dicks, L. M. T. 2018. Bacteria of the genus Xenorhabdus, a novel source of bioactive compounds. Front. Microbiol. 9:3177.

Fuchs, S. W., Grundmann, F., Kurz, M., Kaiser, M., and Bode, H. B. 2014. Fabclavines: Bioactive peptide-polyketide-polyamino hybrids from Xenorhabdus. ChemBioChem 15:512-516.

Fuchs, S. W., Proschak, A., Jaskolla, T. W., Karas, M., and Bode, H. B. 2011. Structure elucidation and biosynthesis of lysine-rich cyclic peptides in Xenorhabdus nematophila. Org. Biomol. Chem. 9:3130-3132.

Garriga, A., Mastore, M., Morton, A., Garcia del Pino, F., and Brivio, M. F. 2020. Immune response of Drosophila suzukiilarvae to infection with the nematobacterial complex Steinernema carpocapsae-Xenorhabdus nematophila. Insects 11:210.

Griffiths-Jones, S. 2003. Rfam: An RNA family database. Nucleic Acids Res. 31:439-441.

Ji, D., Yi, Y., Kang, G.-H., Choi, Y.-H., Kim, P., Baek, N.-I., and Kim, Y. 2004. Identification of an antibacterial compound, benzylideneacetone, from Xenorhabdus nematophila against major plant-pathogenic bacteria. FEMS Microbiol. Lett. 239:241-248.

Lagesen, K., Hallin, P., Rødland, E. A., Stærfeldt, H.-H., Rognes, T., and Ussery, D. W. 2007. RNAmmer: Consistent and rapid annotation of ribosomal RNA genes. Nucleic Acids Res. 35:3100-3108.

Li, B., Kong, L., Qiu, D., Francis, F., and Wang, S. 2021. Biocontrol potential and mode of action of entomopathogenic bacteria Xenorhabdus budapestensis C72 against Bipolaris maydis. Biol. Control 158:104605.
Li, J., Chen, G., and Webster, J. M. 1997. Nematophin, a novel antimicrobial substance produced by Xenorhabdus nematophilus (Enterobactereaceae). Can. J. Microbiol. 43:770-773.

Liu, J., Poinar, G. O., and Berry, R. E. 2000. Control of insect pests with entomopathogenic nematodes: The impact of molecular biology and phylogenetic reconstruction. Annu. Rev. Entomol. 45:287-306.

Lowe, T. M., and Eddy, S. R. 1997. tRNAscan-SE: A program for improved detection of transfer RNA genes in genomic sequence. Nucleic Acids Res. 25: 955-964.

Park, D., Ciezki, K., van der Hoeven, R., Singh, S., Reimer, D., Bode, H. B., and Forst, S. 2009. Genetic analysis of xenocoumacin antibiotic production in the mutualistic bacterium Xenorhabdus nematophila. Mol. Microbiol. 73:938-949.

Rathore, J. S. 2013. Expression, purification, and functional characterization of atypical xenocin, its immunity protein, and their domains from Xenorhabdus nematophila. Int. J. Bacteriol. 2013:1-9.

Shi, Y.-M., and Bode, H. B. 2018. Chemical language and warfare of bacterial natural products in bacteria-nematode-insect interactions. Nat. Prod. Rep. 35:309-335.

Tatusova, T., DiCuccio, M., Badretdin, A., Chetvernin, V., Nawrocki, E. P., Zaslavsky, L., Lomsadze, A., Pruitt, K. D., Borodovsky, M., and Ostell, J. 2016. NCBI prokaryotic genome annotation pipeline. Nucleic Acids Res. 44:6614-6624.

Tobias, N. J., Wolff, H., Djahanschiri, B., Grundmann, F., Kronenwerth, M., Shi, Y.-M., Simonyi, S., Grün, P., Shapiro-llan, D., Pidot, S. J., Stinear, T. P., Ebersberger, I., and Bode, H. B. 2017. Natural product diversity associated with the nematode symbionts Photorhabdus and Xenorhabdus. Nat. Microbiol. 2:1676-1685.

Vozik, D., Bélafi-Bakó, K., Hevesi, M., Böszörményi, E., and Fodor, A. 2015. Effectiveness of a peptide-rich fraction from Xenorhabdus budapestensis culture against fire blight disease on apple blossoms. Not. Bot. Horti Agrobot. Cluj-Napoca 43:547-553.

Xi, X., Lu, X., Zhang, X., Bi, Y., Li, X., and Yu, Z. 2019. Two novel cyclic depsipeptides xenematides $F$ and $G$ from the entomopathogenic bacterium Xenorhabdus budapestensis. J. Antibiot. (Tokyo) 72:736-743.

Zhao, L., Kaiser, M., and Bode, H. B. 2018. Rhabdopeptide/xenortide-like peptides from Xenorhabdus innexi with terminal amines showing potent antiprotozoal activity. Org. Lett. 20:5116-5120. 